\title{
2014 WSGC Elijah High-Altitude Balloon Payload Project
}

\author{
Final Report
}

August 15, 2014

Jadee Kellogg ${ }^{1}$, Madeline Lambert ${ }^{2}$, Danny Ochoa ${ }^{3}$, Evan Schilling ${ }^{3}$, Michael Stefik ${ }^{4}$, Michael Wiznitzer ${ }^{3}$

${ }^{1}$ Ripon College, ${ }^{2}$ University of Wisconsin-La Crosse, ${ }^{3}$ Milwaukee School of Engineering, ${ }^{4}$ University of WisconsinMilwaukee

\begin{abstract}
The purpose of this paper is to discuss the development and findings of the experiments performed on the high altitude balloon payload. After some bonding time in the beginning of the summer, the team got together and decided on the four following experiments: Atmospheric Electric Field, Breakdown Voltage, IR Imaging, and Solar Efficiency/Payload Spin. Subsequently, research and fabrication began which involved learning new skills such as Arduino programming, 3-D modeling, foam cutting, and more. The launch was successful, and the results retrieved were more or less what were expected. This internship opportunity proved to be an amazing learning experience for the team which will be valued for each of their future career experiences.
\end{abstract}

\section{Introduction}

The objective for the team this summer was to design experiments to send up in a high altitude balloon, and successfully collect and analyze data from said experiments. The team was given a budget, size and weight restrictions, and then given free reign as to what they wanted to research. The team decided on four projects: Atmospheric Electric Field, Breakdown Voltage, IR Imaging, and Solar Efficiency/Payload Spin.

\section{Payload Structure}

In order to safely house the experiments, two unique payload structures were designed and constructed. The two capsules, shown in Figure 1, were both constructed with an interior birch aircraft plywood frame and an exterior shell made of polystyrene insulation. The capsules were then wrapped in an aluminized mylar foil which insulates the capsules during the flight.

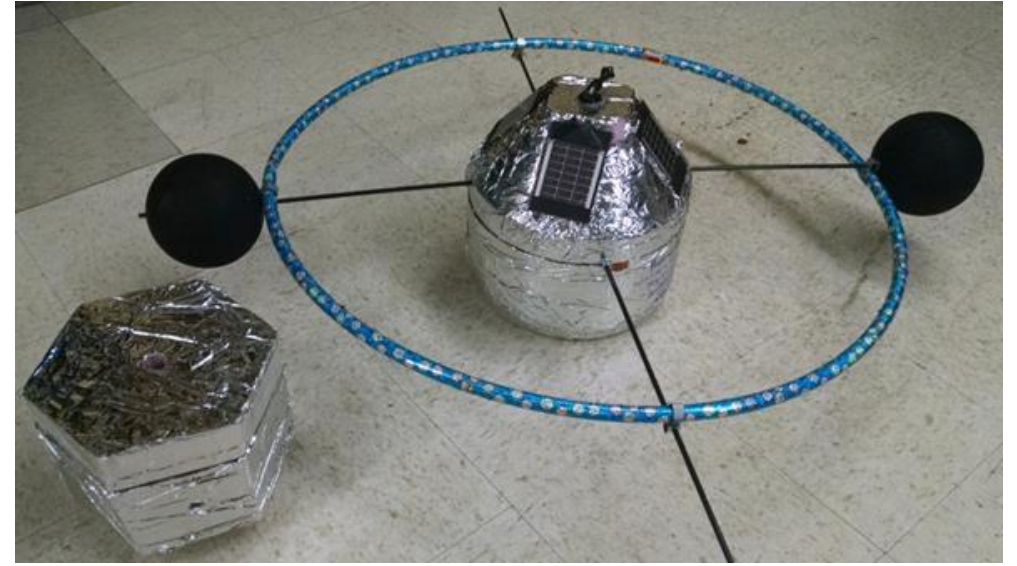

Figure 1: Primary capsule (top right) \& secondary capsule (bottom left) 
The primary capsule had a cylindrical shape with a one foot diameter and was one foot tall. This capsule also featured a passive stabilization ring. For the ring, a forty-eight inch diameter hula hoop was used. Because the hula hoop had a much larger diameter than that of the payload, it significantly increased the moment of inertia of the capsule, which lead to a substantial decrease in the rotational velocity of the payload. The stabilization ring was supported by two fiberglass dowels which also held on the conductive spheres for the electric field experiment.

In order to determine the minimum size of the beams in the frame, an estimation was done using a cantilever beam with a length equal to that of the radius of the payload. The following equation for bending stress was used:

$$
\sigma=\frac{-m \frac{h}{2}}{\frac{1}{12} b h^{3}}
$$

where $m$ is the maximum bending moment (60 in-lb), $\sigma$ is the average maximum stress, in our case 6114.356 psi (Handbook of Finnish Plywood), b is the thickness of the plywood (3/16 in), and $\mathrm{h}$ is the plywood width, which we are solving for. This calculation yields a minimum width of 0.56 inches. Based on this width, the frames for the two capsules were designed. The primary capsule was broken into two halves. The frames for the top and bottom halves are shown in Figure 2 and Figure 3 respectively.

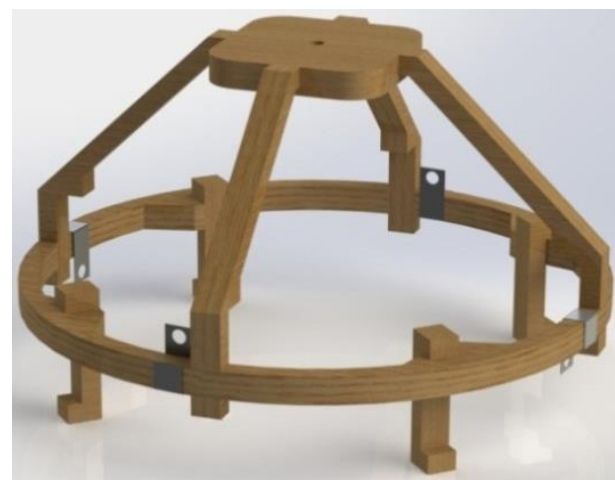

Figure 2: Primary capsule upper frame

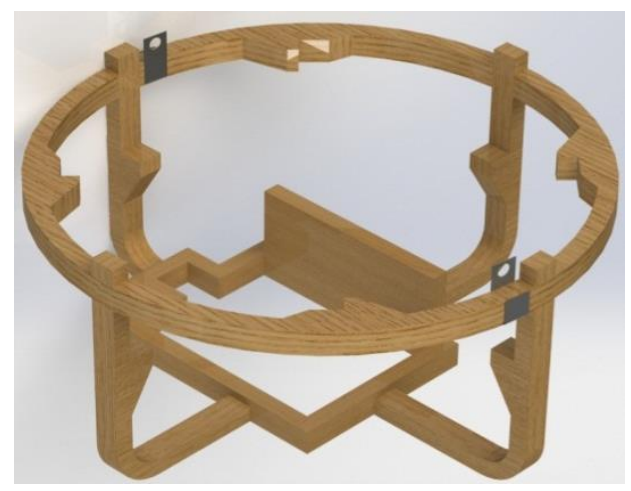

Figure 3: Primary capsule lower frame

The four pegs which protruded down from the upper frame meshed with the extrusions on the ring of the lower frame and held the two halves together. The sheet metal pieces on the rings of the two frames supported the fiberglass dowels that ran through the payload and also served as part of the locking mechanism. Located around the vertical ribs of the frame were "shelve" features that served as an attachment point for the electronics board. The electronics board in the primary payload held all of the electronics for the experiments, as well as a few sensors which recorded the dynamic motion of the payload, as well as atmospheric data.

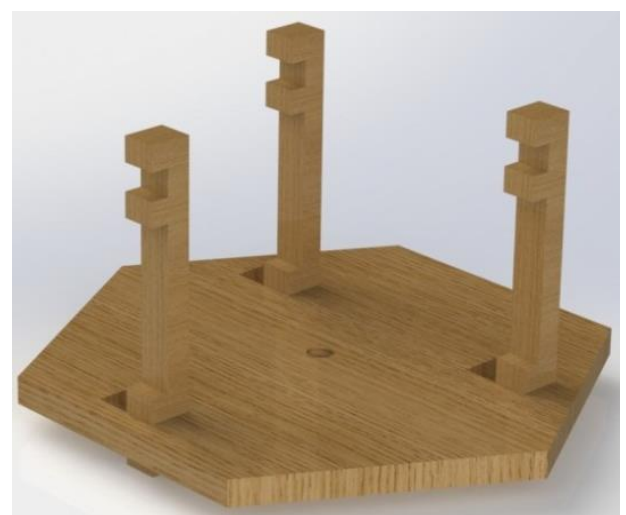

Figure 4: Secondary capsule lower frame

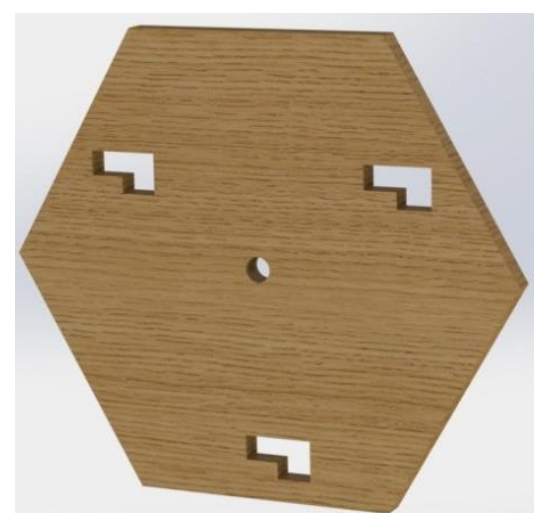

Figure 5: Secondary capsule lid 
The lower frame and lid for the secondary capsule are shown in figure 4 and figure 5 respectively. The platform on the lower section supported the breakdown voltage electronics. The supports protruding out from the platform corresponded with the slots on the lid and locked the two together. A bolt was then run through the hole in the lid and lower section and prevented the two parts from coming apart.

\section{Atmospheric Electric Field Experiment}

Electric fields surround us everywhere. They do many useful things for us in the form of electricity, but are not directly observable through any of our senses. As such, they require specialized equipment to measure and visualize them. Figure 6 shows a depiction of "Earth's Electrical Environment" (National

Figure 6: Schematic of electrical processes in the global electric circuit Research Council, 1986), providing an idea of how unique and varied the electric fields are that exist in our atmosphere.

Purpose: Balloon-borne atmospheric electric field measurements have been taking place since the late 1960s. A common method developed, known and documented as the "double Langmuir probe technique" (R. H. Holzworth and E. A. Bering III, 1998), is used for performing these measurements. Our team sought to replicate the aforementioned method to successfully construct an electric field measuring setup and use it to visualize the field above southern Wisconsin during the balloon flight.

Method: After analyzing an overview of the method, the team set out to design custom hardware in order to build a device capable of performing electric field measurements on our payload. This began with a simple simulation of the technique, which helped us gain insight into how it worked physically. This simulation, performed in the Falstad 2D Electrostatics Applet, is shown on the right in Figure 7.

From this simulation, the team was able to visually see the basic principle of the method: a voltage potential, produced by the electric field forcing a separation of charge, develops across the

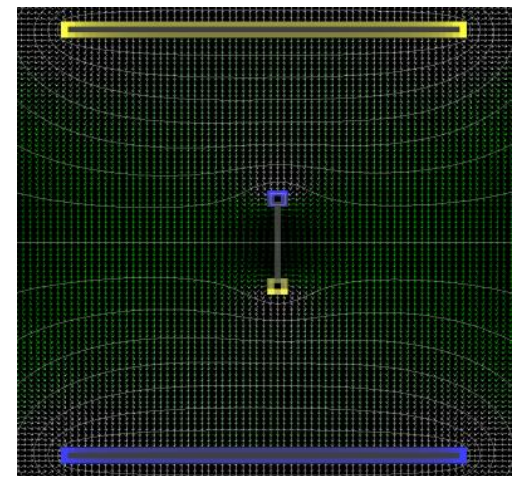

Figure 7: Falstad electric field simulation probes. If a differential voltage measurement is done using an instrument op-amp with the two probes as the positive and negative terminals, a voltage difference that is proportional to the field strength in that direction is obtained. To get a final magnitude value, the voltage difference is simply divided by the separation length, giving us Volts/Meter. The direction can be obtained by watching the rotation of the probes about their vertical axis; whatever the magnetic direction is at the time of a voltage difference maximum, that is the direction of the field (maximums occur when parallel to the electric field gradient, minimums when perpendicular). 


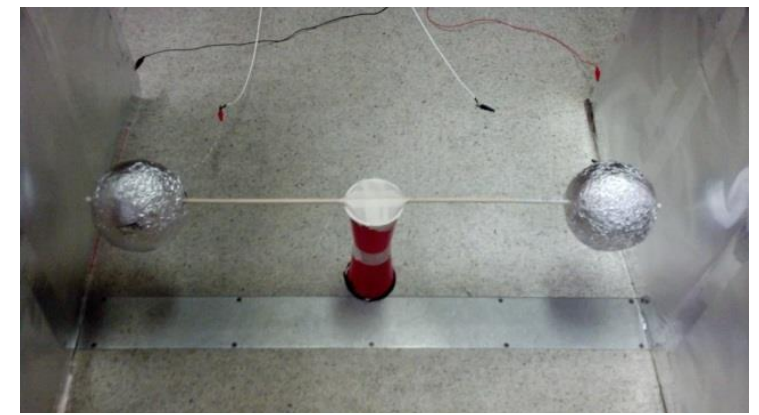

Figure 8: The test setup used to verify the technique
A test setup was then constructed. This consisted of an INA116 as the instrument op-amp, an LM324 used as a $2^{\text {nd }}$ order low-pass filter $(10 \mathrm{~Hz}$ cutoff), two Styrofoam spheres covered in aluminum foil connected by a wooden dowel as the double probes, and two RG188A/U coaxial cables to connect the probes to the instrument op-amp. Two large metal plates $(3 \times 3$ ' area) built using aluminum foil and cardboard were used to generate a test electric

field. This whole setup was placed within a Faraday cage and is pictured above in

Figure 8.

After an external field was applied to the large metal plates using a function generator, a received signal was picked up on the probes and plotted on an oscilloscope. A square wave was successfully received but with ramped planes shown in Figure 9; this was due to the small but noticeable input leakage (advertised on the order of $10^{-15}$ amps but measured in lab to be at $\left.10^{-13} \mathrm{amps}\right)$.

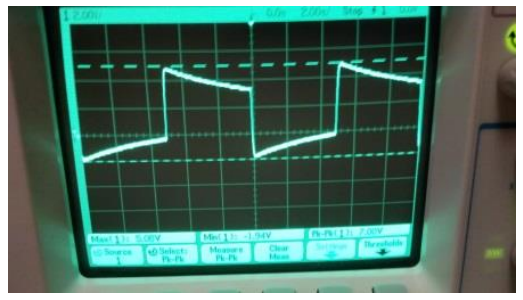

Figure 9: Square wave received on scope

Results: The electronics were placed within a shielded $3 \mathrm{~d}$ printed enclosure; this enclosure was then put inside of the main payload and hooked up to the payload data logger. The final probes were Styrofoam spheres covered with conductive carbon spray separated at 1 meter by a fiberglass boom. The final setup can be seen in Figure 10.

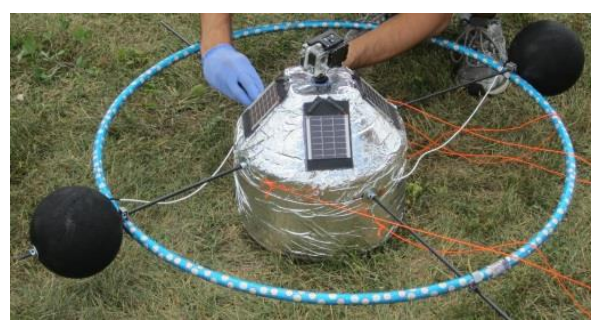

Figure 10: Final payload assembly with field probes

Once the analog data was obtained, a program was written in Python using Enthought Canopy to generate electric field vectors of a certain magnitude at each altitude. Yaw rotation data was taken from the on-board IMU in order to find the direction of the field. Figure 11 shows the final output; the

altitudes are listed in feet, the magnitudes in Volts/Meter, and the direction in magnetic degrees $\left(0^{\circ}\right.$ is magnetic north).

The plot shows we were successful in what we set out to do; a definite trend in the high-altitude data is shown, indicating that a field was detected. The magnitude did not correspond to what we see in scientific papers on the subject, but this gain error could likely be adjusted for future flights to give us more accurate data.

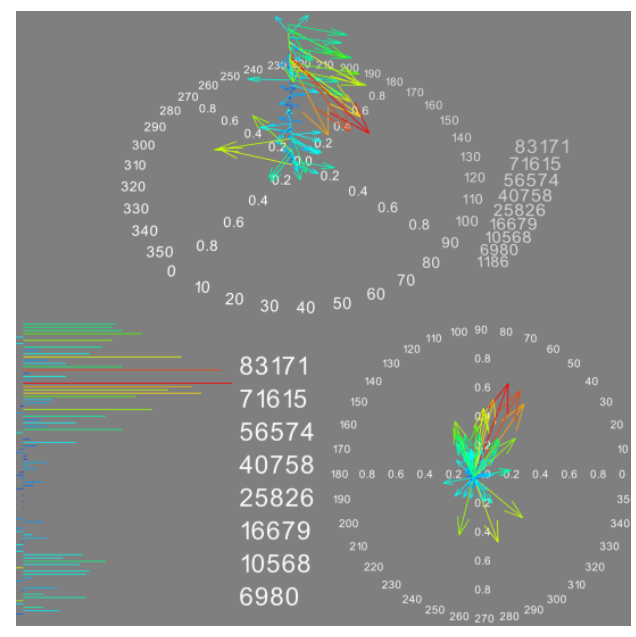

Figure 11: Visualization of the atmospheric electric field 


\section{Breakdown Voltage Experiment}

Purpose: Breakdown voltage is a property innate to insulators. When the correct voltage is applied, the insulator begins to conduct electricity. This is especially important to take into account with high voltage circuits, as sparking may occur between components if the breakdown voltage of air is exceeded between them. The breakdown voltage spark gap experiment was designed to test the effect of the changing atmospheric conditions with the differing altitudes on the breakdown voltage of air.

Method: Using Paschen's Law, $V=$ $\frac{B p d}{(C+\ln (p d))}$, where $\mathrm{p}$ is the pressure in torr, $\mathrm{d}$ is the gap length in centimeters, and $\mathrm{B}$ and $\mathrm{C}$ are constants specific to the different substance, a Paschen's curve was calculated as an estimate for the breakdown voltage of air at high altitude with a half centimeter gap (Figure 12). In order to reach the appropriate voltage, approximately 1000 volts was required. Given the size and weight restrictions, creative measures had to be taken as to how to

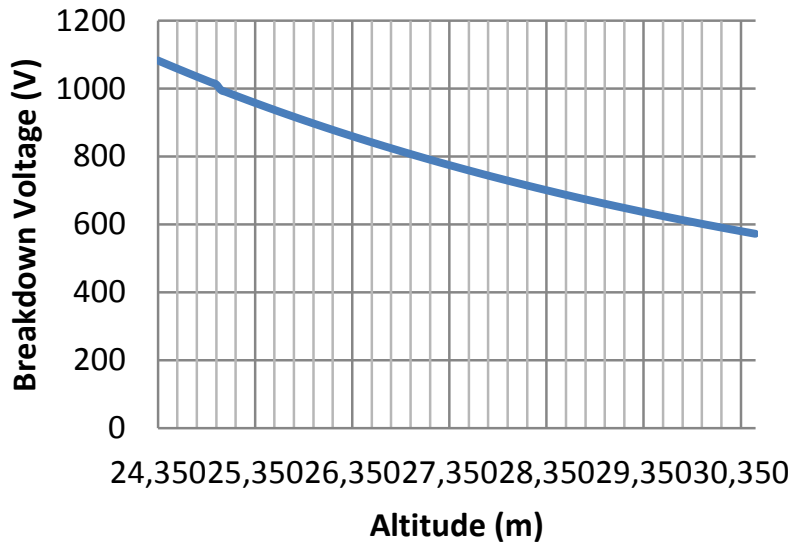

Figure 12: Chart of Breakdown Voltage vs. altitude with a half centimeter gap length produce that voltage. A circuit similar to the one shown in Figure 13 was used, replacing the Geiger tube with the spark gap, and removing the speaker. The spark gap was constructed using two wooden spheres covered in copper tape, mounted on a 3D printed stand. Using a nine volt battery as the power source, a CCFL inverter and voltage doubler were responsible for ramping up the voltage. A digital potentiometer was put into the circuit and controlled via an Arduino nano. It took one minute and 32 seconds to ramp up to the 900 volt maximum, and three minutes and 37 seconds to be brought back down to its minimum voltage, 208. A delay was then set before repeating the cycle. For the first six repetitions of the cycle, this delay was ten minutes. Afterwards, the delay was then set to two minutes. The interrupt function of Arduino coding was utilized so that every time a spark occurred, the time stamp was put into the EEPROM of the nano. In order to protect the nano from a large voltage spike, two resistors were placed in the circuit to bring down the voltage to the manageable five volts maximum. An opto-isolator and an op-amp were also used to further isolate the nano from the high voltage.

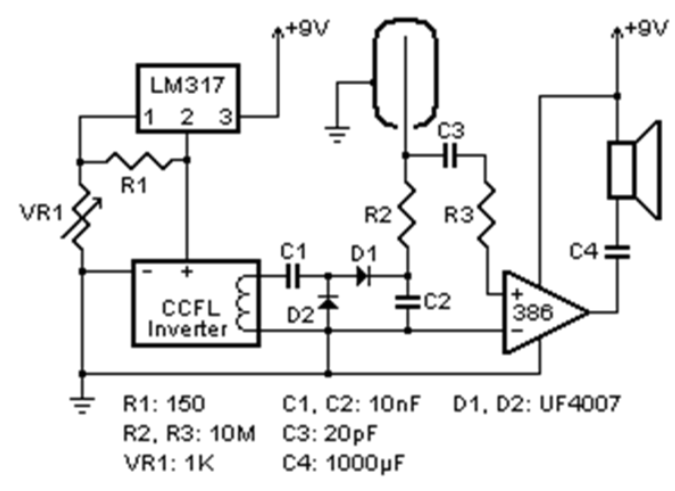

Figure 13: Similar circuit to the one used for the breakdown voltage experiment, found at http://adammunich.com/geigermuller-tubes/ 
Results: Using a separate Arduino code, the EEPROM was read back. It contained one time stamp at 45 minutes and 17 seconds into the flight. After further examination of GPS data (shown in Figure 14), this was found to correspond to an altitude of approximately 28,346 meters, or about 93,000 feet. Using Paschen's Law and the pressure data from the payload electronics, the calculated breakdown voltage at the time the spark occurred was 848.6 volts.

Altitude vs. Time

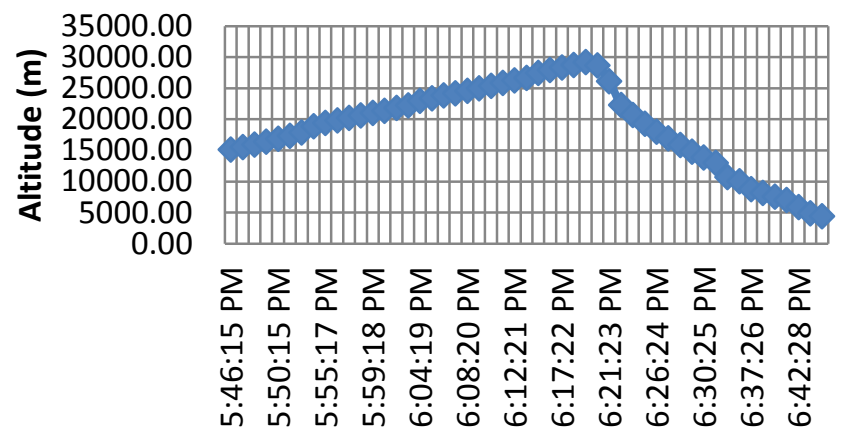

Figure 14: Graph of GPS data collected by secondary tracking payload
The actual voltage at this time was 840 . This could be due to humidity or moisture entering the payload container, or various other environmental factors.

One spark is not enough to draw reliable or precise conclusions. If the experiment were flown again, the delay time could be shortened, as could the gap length, to allow for more sparks to occur. However, the one data point retrieved did correlate with the predictions made, which is promising for any future attempts at recreating the experiment.

\section{Infrared Imaging}

Purpose: The Infrared (IR) imaging project was developed with the goal of analyzing plant and crop health at various altitudes. This experiment was conducted using a Canon camera which utilized special software to take time lapse photos. An external filter system was also built with both visible and IR imaging filters. Crop health can be tracked after the IR and visible light images are combined to form a Normalized Difference Vegetation Index (NDVI) picture.

Method: The initial step of the project was working with the Canon A1200 camera. The Canon A1200 was chosen because the other camera possibility, the Canon A495, failed to properly allow the desired time lapse function to work. The Canon A1200 cooperated with the preferred method, the Canon Hacker Development Kit (CHDK) software, and the software was successfully downloaded onto it, in order to give the camera time lapse functionality. CHDK was programed to take pictures every four seconds for the duration of the flight. The camera also had its internal IR blocking filter removed so the external filters could be used. The two filters, visible and infrared, were designed to be mounted externally from the camera and controlled by a Servo motor using the Arduino Mega. A camera housing was designed to have three main functions: to securely hold the camera, to serve as an attachment point for the servo motor, which was mounted externally from the main housing using a sheet metal bracket and small bolts to firmly hold it in place, and to house and protect the filters, which were mounted into a filter switching mechanism that slid within the camera housing. This mechanism functioned using two arms; one connected to the Servo, and the other connected to the filter mount. After watching how the Servo rotated the mechanism, the proper initial and final angles were chosen. Both the camera housing and the filter switching mechanism were 3D printed using the MakerBot 2X. When ready to run the experiment, CHDK and the filter switching mechanism were manually 
synced. Electronically syncing up the Arduino and Servo with the CHDK software was researched extensively and there was no practical method to align both systems in time. As added protection, four hand warmers were included in the payload to keep the camera warm enough to take pictures throughout its entire flight.

Final data and results: On launch day, while other balloon preparations were being made, the four hand warmers were attached to the payload's interior frame. When the electronics were ready to be turned on, the Servo was activated and the camera's CHDK program was manually synced with the rotations. Once finished with the camera preparations, the payload was then passed along so other experiments could be initiated. This is believed to be the cause of our malfunctioning filter switching mechanism. Since no visible light images were obtained throughout the flight, the mechanism must have become blocked with debris while sitting on the ground before the launch. In order to use the data we had gotten, in the form of IR pictures, Google Earth Images were used as the comparable visible light picture. This, of course, is not an accurate method to analyze the plant health in the area of Mt. Horeb on the day of the launch. However, as the Google Earth Images used were only a couple months old, the NDVI pictures are a representation of the expected results. Below, Figure 15, is an IR picture our camera took during flight. The picture on the right, Figure 16, is the visible light picture taken from Google Earth. After combining the two pictures using the proper formula, in a photo editing program named Gimp, we came up with the NDVI picture, Figure 17.

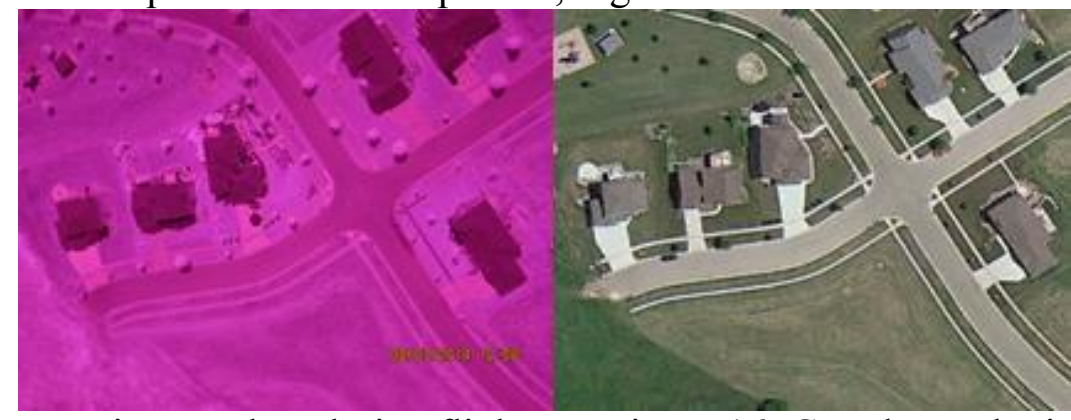

Figure 15: IR picture taken during flight Figure 16: Google Earth picture

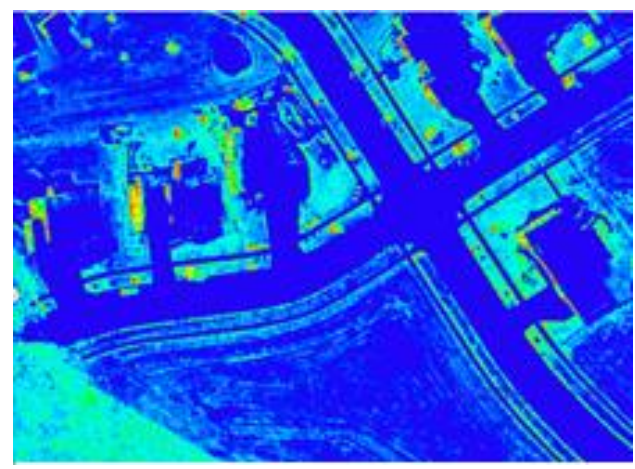

Figure 17: NDVI picture

The trees along the edge of the street are a yellow/orange. Some people's yards are a brighter blue than others. The idea is that the warmer the colors, the healthier the area should be. The houses and the concrete are a dark blue because they do not produce any heat. Although it cannot be confirmed that these results are $100 \%$ accurate, the general concept was proved. 


\section{Solar Cell Experiment}

Purpose: As technology becomes more advanced, much research is put into energy conservation so that people can save money and the world can become a greener place. One such method is the use of solar cells to convert the sun's light into usable energy. The goals of this experiment were to explore how efficient solar cells are in this converting process relative to altitude and temperature and also how effectively they can be used to measure rotation of an object.

Method: Efficiency is calculated by dividing the solar cell output power by the amount of the sun's light that is hitting the solar cell, as described in the equation: $\eta=\frac{P_{\text {output }}}{P_{\text {input } * \text { Area }}}$ (Honsberg \& Bowden). As these particular solar cells used for the experiment were $73 \times 74 \mathrm{~mm}$ rated $3.6 \mathrm{~V}$ at 50 $\mathrm{mA}$, it was calculated that the maximum rated power they could produce was 0.18 watts, for an efficiency of about $3.33 \%$, assuming a resistance of $72 \mathrm{ohms}$ and that the sun's intensity was 1000 watts $/ \mathrm{m}^{2}$ on Earth's surface. This resistance was calculated by dividing the rated current by the rated voltage using Ohm's law and was also verified experimentally to be the optimal resistance where the solar cells produced the maximum power. Due to the molecular processes that occur in a solar cell, it was hypothesized that solar cells would become more efficient at colder temperatures, which means that the efficiency should peak between 50-60,000 feet in Earth's atmosphere where it's the coldest, almost negative $70^{\circ} \mathrm{C}$ (Solar Cell Efficiency, 2009). It was also assumed that the efficiency would generally increase with altitude, since there would be less sunlight absorption the higher up in Earth's atmosphere (Andrew, 2011). It should be noted the sun's intensity was always assumed to be 1,000 watts $/ \mathrm{m}^{2}$ regardless of altitude, so it was only presumed the efficiency would increase due to higher power output of the solar cells. This might not actually reflect the actual efficiency of the solar cells, since even though the power output could be higher, the efficiency could nevertheless still be lower. For future endeavors, the solar irradiance should also be measured with altitude.

The spinning behavior of the payload was determined by mounting four solar cells, one in each direction, on the payload at $45^{\circ}$ angles. This angle was chosen for two reasons. One is the fact the efficiency of the solar cell is greater when placed in the most optimum angle towards the sun (which in August in Madison is about $55^{\circ}$ from the vertical at solar noon which was near the time of the launch) (Solar Angle Calculator, 2014). The other was so that the solar cells would be sufficiently angled enough that only one of the four solar cells would have the maximum output voltage at any given time. The idea would be then to keep track which of the four solar cells had the maximum output voltage, which would then be able to be processed to output if the payload was turning counterclockwise or clockwise. Additionally, a graph of the rate of rotation would also be generated and compared to the one generated by the IMU (Inertial Measurement Unit) for comparison to see if they looked the same as was hypothesized.

Final data and results: The flight was fairly successful, and the hypotheses for the solar experiments were confirmed for the most part. The efficiency results are shown below in Figure 18 and were calculated by only pulling the power values generated from the solar cell that was directly facing the sun during the flight (the cell that had the highest power value of the four solar cells). 


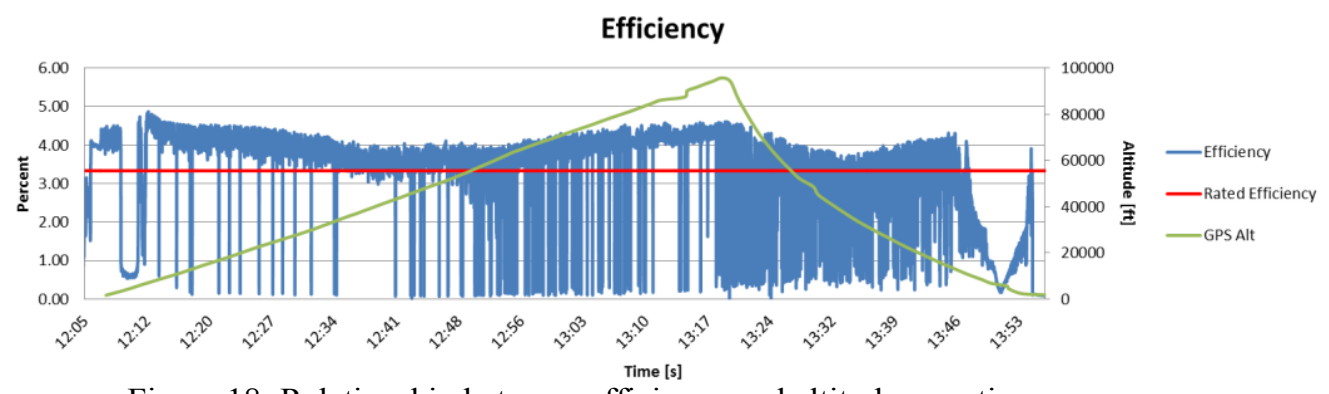

Figure 18: Relationship between efficiency and altitude over time

The red line symbolizes the rated efficiency of the solar cells which was at around $3.33 \%$. As can be clearly seen, the efficiency was above that for the entire flight, ranging between 4 and $4.8 \%$. It peaked near 7,000 ft. at 4.87\%, when the payload reached its highest altitude at around 96,000 ft. at $4.61 \%$ (which makes sense due to higher sunlight intensity), and also at $14,000 \mathrm{ft}$. at $4.31 \%$ but not at the other expected range between 50 and 60,000 feet. Unfortunately, the temperature sensor output skewed results because of the hand warmers inside the payload, so the data could not be used to see if the temperature was actually colder at those altitudes. One theory could be the fact that the optimum solar angle mentioned before was not constant throughout the flight and was at its optimum only when the payload reached 7,000 ft. on the way up and 14,000 ft. on the way down. To get more accurate results, a solar tracker can be used to keep the solar cell at the optimum angle towards the sun, and the actual temperature of the solar cell can be monitored as well.

The other experiment proved to be an effective method of measuring payload spin and rate of rotation. The results are shown below in Figures 19-21:
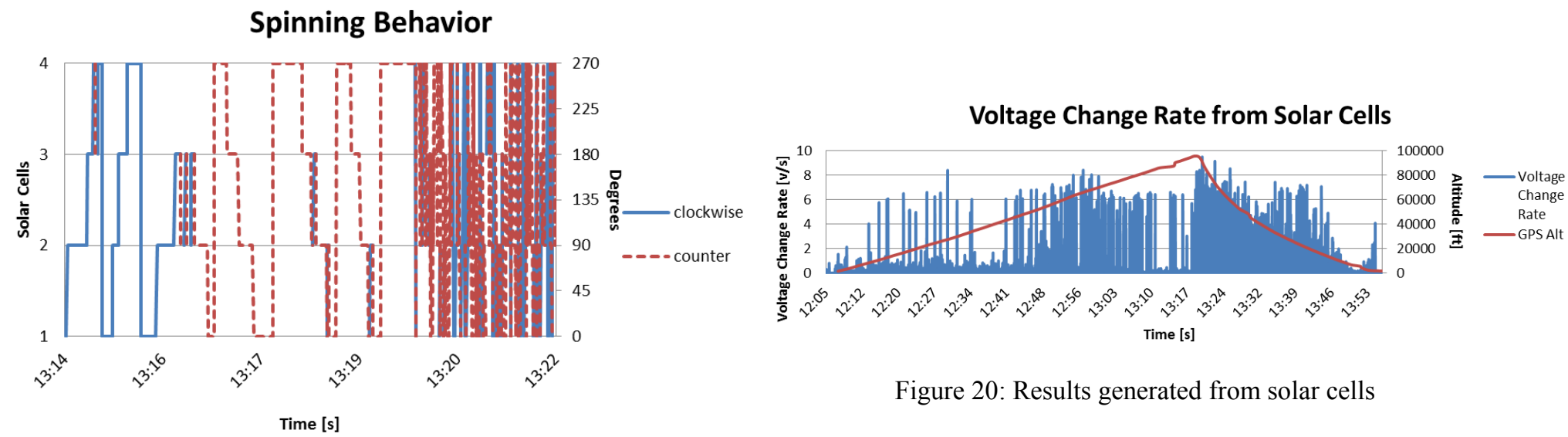

Figure 20: Results generated from solar cells

Figure 19: Solar cell spinning behavior

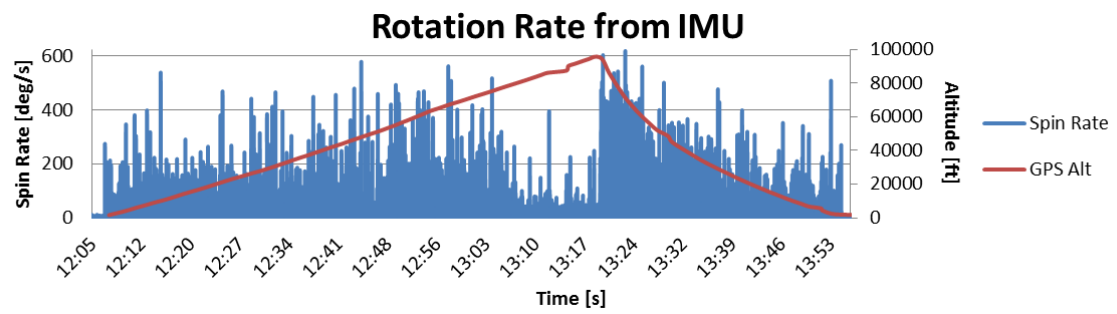

Figure 21: Results generated from IMU

As shown above in Figure 19, one can see at what points the payload turned and if it was turning counterclockwise or clockwise. It can also be observed that the balloon popped at 1:19pm because that is when it begins to spin rapidly. Figures 20-21 show that the rate graphs generated 
from the solar cells and the IMU are very similar, proving that using solar cells to measure rotation rate is a feasible method.

\section{Conclusions}

Each of the separate experiments achieved their objectives. With the specified alterations to each part of the project, better and more accurate results could be obtained with another launch. All in all, the project went well, with all the team members collaborating to ensure its success.

\section{References}

Andrew. (2011, March 2). "Natural Frequency." Retrieved August 7, 2014, from Solar Radiation: http://naturalfrequency.com/wiki/solar-radiation

“Enthought: Scientific Computing Solutions," Enthought, Retrieved July 2014, from https://www.enthought.com/products/canopy/

Falstad, P., "Falstad Math and Physics: EM Static Applet," Retrieved July 2014, from http://www.falstad.com/emstatic/index.html

Handbook of Finnish Plywood, http://www.upm.com/cn/products/plywood/Documents/Handbook_EN.pdf

Holzworth, R.H. \& Bering III, E. A., "Ionospheric Electric Fields From Stratospheric BalloonBorne Probes," Measurement Techniques in Space Plasmas: Fields, vol. 103, Washington, SC, American Geophysical Union, 1998, pp. 79-84.

Honsberg, C., \& Bowden, S. (n.d.). "PV Education.” Retrieved July 6, 2014, from PVeducation.org: http://pveducation.org/

"Insulator Breakdown Voltage." All About Circuits. Retrieved June 2014, from http://www.allaboutcircuits.com/vol_1/chpt 12/8.html

Munich, Adam. (2012). “Geiger-Müller Tubes.” Retrieved June 2014, from http://adammunich.com/geiger-muller-tubes/

National Research Council, The Earth's Electrical Environment, Washington, DC: The National Academies Press, 1986.

"Paschen's Law." Retrieved June 2014 from http://www.donbot.com/Enerbot/LENR/LENR5Engine/LEN019PaschenzLaw.html

Solar Angle Calculator. (2014). Retrieved July 6, 2014, from Solar Electricity Handbook: http://solarelectricityhandbook.com/solar-angle-calculator.html

Solar Cell Efficiency. (2009). Retrieved July 6, 2014, from Solar Power information: http://www.solarpower2day.net/solar-cells/efficiency/ 